\title{
Resolução numérica das equações de Navier-Stokes usando o método das interfaces imersas com correção do salto implícita em malha deslocada
}

\author{
Gabriela A. dos Reis, \\ Italo V. M. Tasso, \\ Leandro F. de Souza, José A. Cuminato. \\ Depto de Matemática Aplicada e Estatística, ICMC, USP, \\ 13566-590, São Carlos, SP \\ E-mail: gareis@icmc.usp.br, italo@tasso.com.br, lefraso@icmc.usp.br, jacumina@icmc.usp.br.
}

\begin{abstract}
Resumo: $O$ método das interfaces imersas (MII) tem sido utilizado para simular problemas de escoamento de fluidos em geometrias complexas. Uma das dificuldades do MII é calcular os saltos nos valores da função e em suas derivadas, pois nem sempre esses valores são conhecidos analiticamente. Em [4], foi desenvolvido um MII que calcula numericamente os saltos, por meio de um processo iterativo explícito que utiliza os pontos vizinhos da descontinuidade. Seguindo essa ideia, foi desenvolvida em [5] uma versão implícita não-iterativa desse método para resolver uma equação de Poisson. O presente trabalho apresenta a resolução das equações de NavierStokes, pelo método da projeção, usando o MII desenvolvido em [5] em variáveis primitivas e em uma malha deslocada.
\end{abstract}

Palavras-chave: Navier-Stokes, malha deslocada, interface imersa, métodos de alta ordem.

\section{Introdução}

O método das interfaces imersas (MII) tem sido empregado em simulações de escoamento de fluidos em geometrias altamente complexas, que são frequentemente encontradas em muitos campos da ciência e engenharia. Esse método permite a imposição de uma grande variedade de condições de contorno [8], incluindo superfícies livres, interfaces fluido-fluido, fluido-sólido, etc. A principal vantagem da técnica de interface imersa está na alta ordem de aproximação na modelagem de geometrias complexas, sem a necessidade de transformação de coordenadas, necessitando simplesmente de uma malha cartesiana uniforme.

O MII-analítico (MII-A) foi proposto por [3] e então permitia obter segunda ordem de precisão. Com o MII-explícito (MII-E) [4], quarta ordem foi obtida na simulação das equações de Navier-Stokes utilizando a formulação corrente-vorticidade. Os valores dos saltos no valor da função e em suas derivadas, necessários para o MII, são calculados numericamente, por meio de um processo iterativo explícito, ao invés de analiticamente como na versão original.

O presente trabalho apresenta o MII-implícito (MII-I), construído a partir do MII-E, com algumas modificações. O MII-E tem um inconveniente que é a utilização de processos iterativos para a estimativa dos valores dos saltos na resolução da equação de Poisson. O sistema linear tem que ser resolvido várias vezes. A cada iteração, o valor do salto é atualizado até convergir. Isso gera um processo caro computacionalmente. Portanto, é proposto o MII-I, uma versão do MII-E que trata as condições de salto de forma implícita, diretamente como parte da solução do sistema linear. Isso permite resolver o sistema linear uma única vez, proporcionando um ganho significativo em tempo de processamento. Além disso, o presente trabalho resolve as equações de Navier-Stokes em uma malha deslocada com variáveis primitivas, ao contrário do MII-E que usava a formulação corrente-vorticidade com malha colocalizada.

O método das soluções manufaturadas é utilizado nos testes de análise de convergência. 


\section{Equações governantes}

A dinâmica de um escoamento Newtoniano incompressível descrito pelas equações de NavierStokes é dada por

$$
\begin{aligned}
\frac{\partial \mathbf{u}}{\partial t}+\mathbf{u} \cdot \nabla \mathbf{u} & =-\nabla p+\nu \nabla^{2} \mathbf{u}+\mathbf{g} \\
\nabla \cdot \mathbf{u} & =0
\end{aligned}
$$

onde $\nu$ é viscosidade cinemática, $\mathbf{u}=(u, v)$ o vetor velocidade, $p$ a pressão sobre densidade constante e g o campo gravitacional. No presente trabalho, as condições de contorno adotadas são do tipo Dirichlet para a velocidade e Neumann homogênea para a pressão. O método de Euler explícito é usado para avançar (1) no tempo. O sistema de EDPs (1) e (2) é resolvido utilizando a versão incremental do método da projeção [1].

\section{Método numérico}

A Figura 1 ilustra o domínio que será utilizado para simular a solução das equações (1) e (2). A malha é gerada de forma que o contorno rígido seja representado por uma interface imersa. A solução em $\Omega$ é de interesse, fora disso é considerada nula. É importante notar que a interface não coincide com pontos de malha.

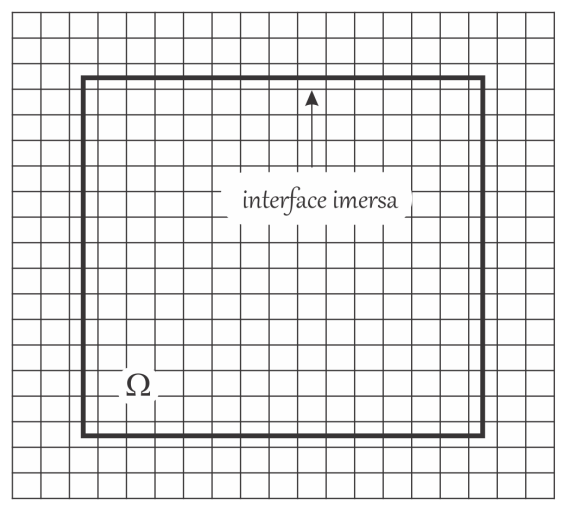

Figura 1: Ilustração do domínio de interesse nesse trabalho. O contorno rígido é tratado como uma interface imersa, e esse não coincide com pontos de malha.

\subsection{Método das interfaces imersas}

O MII-I, apresentado neste trabalho, é inspirado nas ideias presentes em [4]. Os autores desenvolveram o MII-E para resolver as equações de Navier-Stokes utilizando a formulação correntevorticidade. No MII-E, as condições de salto são calculadas numericamente por meio de um processo iterativo explícito e quarta ordem é obtida. A seguir são apresentadas as ideias desse método.

Considere uma função $f(x)$ com uma descontinuidade no ponto $x=x_{\alpha}$. Esse ponto está entre os pontos de malha $x_{i}$ e $x_{i+1}$. Ao se realizar cálculos no ponto $x_{i}$, o valor de $f\left(x_{i+1}\right)$ não pode ser usado diretamente, devido à presença da descontinuidade. Deve-se extrapolar um valor para $f\left(x_{i+1}\right)$. O MII usa uma expansão em série de Taylor no ponto $x_{\alpha}$ para aproximar $f(x)$ no ponto $x_{i+1}$.

Assumimos que $f(x)$ seja analítica em todos os pontos do domínio $D=\left\{x \mid x_{i} \leq x \leq x_{i+1}\right\}$, exceto no ponto $x_{\alpha}$ onde há um salto (descontinuidade) no valor da função ou suas derivadas. A expansão em série de Taylor padrão em torno de $x_{i}$ não pode ser usada para aproximar $f\left(x_{i+1}\right)$, 
a menos que um termo de correção $J_{\alpha}$ seja adicionado:

$$
f\left(x_{i+1}\right)=f\left(x_{i}\right)+f^{(1)}\left(x_{i}\right) h+f^{(2)}\left(x_{i}\right) \frac{h^{2}}{2 !}+\cdots+J_{\alpha},
$$

onde

$$
J_{\alpha}=\left[f^{(0)}\right]_{\alpha}+\left[f^{(1)}\right]_{\alpha}\left(h^{+}\right)+\frac{1}{2 !}\left[f^{(2)}\right]_{\alpha}\left(h^{+}\right)^{2}+\frac{1}{3 !}\left[f^{(3)}\right]_{\alpha}\left(h^{+}\right)^{3}+\cdots+\frac{1}{n !}\left[f^{(n)}\right]_{\alpha}\left(h^{+}\right)^{n},
$$

$h=x_{i+1}-x_{i}$ e $h^{+}=x_{i+1}-x_{\alpha}$. O termo $\left[f^{(n)}\right]_{\alpha}$ representa o salto no valor da $n$-ésima derivada da função em $x=x_{\alpha}$, isto é,

$$
\left[f^{(n)}\right]_{\alpha}=\lim _{x \rightarrow x_{\alpha}^{+}} f^{(n)}(x)-\lim _{x \rightarrow x_{\alpha}^{-}} f^{(n)}(x) .
$$

Usando o termo de correção $J_{\alpha}$, pode-se modificar qualquer método de diferenças finitas, e o método corrigido pelo salto deverá manter a ordem de precisão do original quando o estêncil envolver uma singularidade ou salto na função.

\subsubsection{Discretização espacial}

As derivadas presentes em (1) e (2) serão discretizadas utilizando o método de diferenças finitas compactas de quarta ordem [2]. Na presença de descontinuidades, uma aproximação para a primeira derivada pode ser escrita como

$$
L_{i-1}^{1} f_{i-1}^{(1)}+L_{i}^{1} f_{i}^{(1)}+L_{i+1}^{1} f_{i+1}^{(1)}=R_{i-1}^{1} f_{i-1}+R_{i}^{1} f_{i}+R_{i+1}^{1} f_{i+1}+\left(L_{I}^{1} J_{\alpha 1}-R_{I}^{1} J_{\alpha 0}\right),
$$

e para a segunda derivada,

$$
L_{i-1}^{2} f_{i-1}^{(2)}+L_{i}^{2} f_{i}^{(2)}+L_{i+1}^{2} f_{i+1}^{(2)}=R_{i-1}^{2} f_{i-1}+R_{i}^{2} f_{i}+R_{i+1}^{2} f_{i+1}+\left(L_{I}^{2} J_{\alpha 2}-R_{I}^{2} J_{\alpha 0}\right),
$$

onde $L_{i}^{n}$ e $R_{i}^{n}$ são, respectivamente, os coeficientes dos lados esquerdo e direito da aproximação para a $n$-ésima derivada e $J_{\alpha n}$ são as expansões dos saltos em série de Taylor de $f^{(n)}$ no ponto $x=x_{\alpha} . I=i+1$ ou $I=i-1$ dependendo da posição da interface. Observe que $J_{\alpha 1}, J_{\alpha 2}$ são as expansões dos saltos em relação a derivada (valor desconhecido) e $J_{\alpha 0}$ em relação a função (valor conhecido).

Em uma malha colocalizada, para o cálculo de $J_{\alpha n}$ como em (4), o valor de $\left(h^{+}\right)$é sempre o mesmo. No entanto, em uma malha deslocada, dois casos devem ser considerados, como visto na Figura 2.
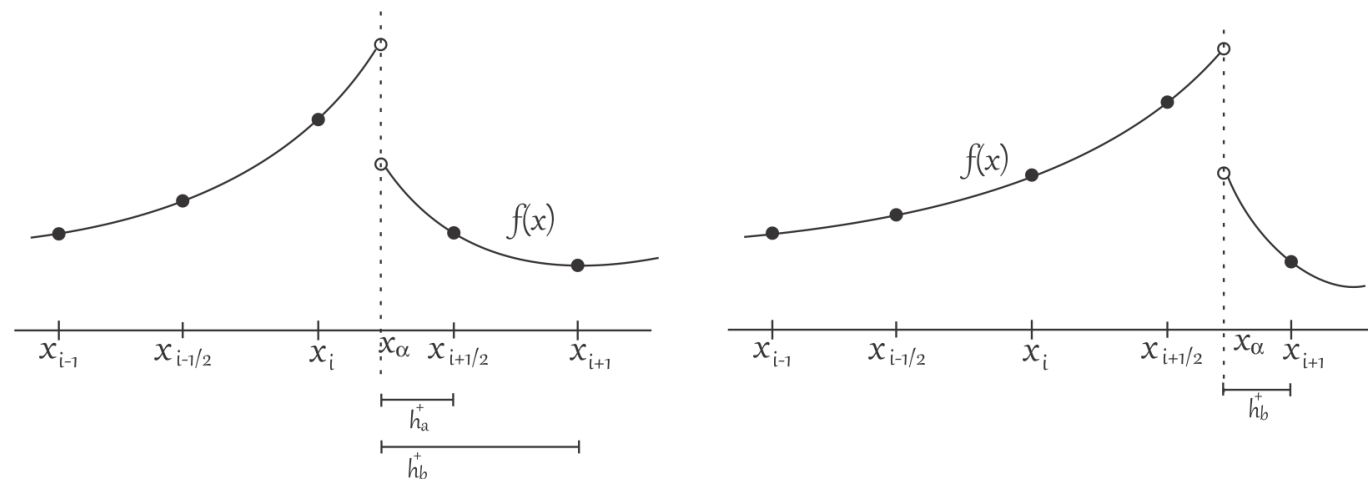

Figura 2: Casos possíveis onde a interface intersecta a malha. Na esquerda, tanto a quantidade conhecida (valor da função) quanto a desconhecida (derivadas) estão depois da descontinuidade. $\mathrm{Na}$ direita, apenas a quantidade desconhecida está depois da descontinuidade.

No caso descrito na Figura 2, as quantidades desconhecidas (primeira ou segunda derivada) estão nos pontos com índices inteiros $(i, i+1, \cdots)$. As quantidades conhecidas (valor da função) 
estão nos pontos com índices semi-inteiros $(i+1 / 2, i+3 / 2, \cdots)$. Quando tanto a quantidade conhecida quanto a desconhecida estão depois da descontinuidade, são necessários dois $J_{\alpha n}$, cada um com seu valor apropriado para $\left(h^{+}\right)$. Esse caso pode ser visto no lado esquerdo da Figura 2. Se apenas a quantidade desconhecida estiver depois da descontinuidade, apenas um valor de $J_{\alpha n}$ é necessário, como pode ser visto no lado direito da Figura 2.

Os saltos são definidos como

$$
\left[f^{(n)}\right]_{\alpha}=f_{+}^{(n)}-f_{-}^{(n)},
$$

em que $f_{+}^{(n)}$ e $f_{-}^{(n)}$ podem ser obtidos numericamente por

$$
\begin{aligned}
& f_{+}^{(n)}=c_{n_{\alpha}} f_{\alpha}+c_{n_{i+2}} f_{i+2}+c_{n_{i+3}} f_{i+3}+c_{n_{i+4}} f_{i+4}+c_{n_{i+5}} f_{i+5}+c_{n_{i+6}} f_{i+6}, \\
& f_{-}^{(n)}=c_{n_{\alpha^{-}}} f_{\alpha}+c_{n_{i-1}} f_{i-1}+c_{n_{i-2}} f_{i-2}+c_{n_{i-3}} f_{i-3}+c_{n_{i-4}} f_{i-4}+c_{n_{i-5}} f_{i-5} .
\end{aligned}
$$

Note que, os pontos $x_{i}$ e $x_{i+1}$ foram evitados para contornar problemas de instabilidade. Essa aproximação possui quarta ordem de precisão.

Os coeficientes $c_{n}$ para calcular

$$
f_{\alpha}^{(n)}=c_{\alpha} f_{\alpha}+c_{i} f_{i}+c_{i+1} f_{i+1}+c_{i+2} f_{i+2}+c_{i+3} f_{i+3}+c_{i+4} f_{i+4},
$$

são obtidos da resolução do sistema linear

$$
\left[\begin{array}{cccccc}
1 & 1 & 1 & 1 & 1 & 1 \\
0 & h_{i} & h_{i+1} & h_{i+2} & h_{i+3} & h_{i+4} \\
0 & h_{i}^{2} & h_{i+1}^{2} & h_{i+2}^{2} & h_{i+3}^{2} & h_{i+4}^{2} \\
0 & h_{i}^{3} & h_{i+1}^{3} & h_{i+2}^{3} & h_{i+3}^{3} & h_{i+4}^{3} \\
0 & h_{i}^{4} & h_{i+1}^{4} & h_{i+2}^{4} & h_{i+3}^{4} & h_{i+4}^{4} \\
0 & h_{i}^{5} & h_{i+1}^{5} & h_{i+2}^{5} & h_{i+3}^{5} & h_{i+4}^{5}
\end{array}\right]\left[\begin{array}{c}
c_{\alpha} \\
c_{i} \\
c_{i+1} \\
c_{i+2} \\
c_{i+3} \\
c_{i+4}
\end{array}\right]=\left[\begin{array}{c}
1 \delta_{n 0} \\
1 \delta_{n 1} \\
2 ! \delta_{n 2} \\
3 ! \delta_{n 3} \\
4 ! \delta_{n 4} \\
5 ! \delta_{n 5}
\end{array}\right],
$$

em que $h_{i}=x_{i}-x_{\alpha}$ e $\delta_{i j}$ é a função delta de Kronecker

$$
\delta_{i j}=\left\{\begin{array}{ll}
1 & i=j \\
0 & i \neq j
\end{array} .\right.
$$

\subsection{Equação de Poisson}

Ao resolver a equação de Poisson,

$$
\nabla^{2} f=g,
$$

obtida no método da projeção, a equação (7) torna-se

$$
R_{i-1}^{2} f_{i-1}+R_{i}^{2} f_{i}+R_{i+1}^{2} f_{i+1}=L_{i-1}^{2} f_{i-1}^{(2)}+L_{i}^{2} f_{i}^{(2)}+L_{i+1}^{1} f_{i+1}^{(2)}-\left(L_{I}^{2} J_{\alpha 2}-R_{I}^{2} J_{\alpha 0}\right) .
$$

Nesse caso, o valor da segunda derivada $\left(f^{(2)}\right)$ é conhecido e o valor da função $(f)$ passa a ser o desconhecido. Note que o termo de correção é adicionado no lado direito da equação, como proposto pelo MII-E [4]. Uma solução, adotada pelos autores, é começar com um valor tentativo para $f$ e melhorá-lo iterativamente

$$
f=\beta f_{\text {new }}+(1-\beta) f .
$$

Uma desvantagem dessa formulação é que se trata de um processo iterativo. O parâmetro de relaxação $\beta$ influencia na convergência e é calibrado manualmente para cada problema. Uma escolha ruim pode significar uma convergência lenta ou não convergência.

Uma alternativa é calcular as correções como parte da solução diretamente. Assim, a equação a ser resolvida passa a ser

$R_{i-1}^{2} f_{i-1}+R_{i}^{2} f_{i}+R_{i+1}^{2} f_{i+1}+\left(L_{I}^{2} J_{\alpha 2}^{*}-R_{I}^{2} J_{\alpha 0}^{*}\right)=L_{i-1}^{2} f_{i-1}^{(2)}+L_{i}^{2} f_{i}^{(2)}+L_{i+1}^{1} f_{i+1}^{(2)}-\left(L_{I}^{2} J_{\alpha 2}^{* *}-R_{I}^{2} J_{\alpha 0}^{* *}\right)$. 
As condições de salto $J_{\alpha 2}, J_{\alpha 0}$ podem ser incorporadas na matriz do sistema linear, pois elas são aproximadas como funções lineares dos valores discretos de $f$. O termo $J_{\alpha n}^{*}$ envolve valores desconhecidos de $f$, geralmente dentro do domínio, e o termo $J_{\alpha n}^{* *}$ envolve valores conhecidos de $f$, geralmente condições de contorno. Esse é o MII-I [5].

\section{Resultados}

A análise dos resultados é feita por meio do método das soluções manufaturadas [6]. No primeiro caso, é mostrada a análise de convergência no cálculo das derivadas ao utilizar o método das interfaces imersas de quarta ordem apresentado anteriormente (MII-I). No segundo caso, é simulado o escoamento em uma cavidade. Em ambos os casos, é utilizada uma malha deslocada, onde a pressão está no centro da célula e as velocidades nas arestas.

\subsection{Cálculo das derivadas}

Sejam as soluções prescritas

$$
U=\sin (2 \pi x) \sin (2 \pi y), \quad V=\cos (2 \pi x) \cos (2 \pi y), \quad P=\sin (2 \pi x) \cos (2 \pi y),
$$

no domínio $0 \leq x, y \leq 1$. O contorno está descolado de $\frac{1}{3} h$ em relação aos pontos de malha, portanto não passa pelos pontos onde são amostradas as velocidades ou pressão. $h$ é a largura da célula. A Figura 3 mostra a evolução dos erros absolutos na norma $L_{\infty}$ com o refinamento da malha. Observe que todas as derivadas convergem com ordem 4.

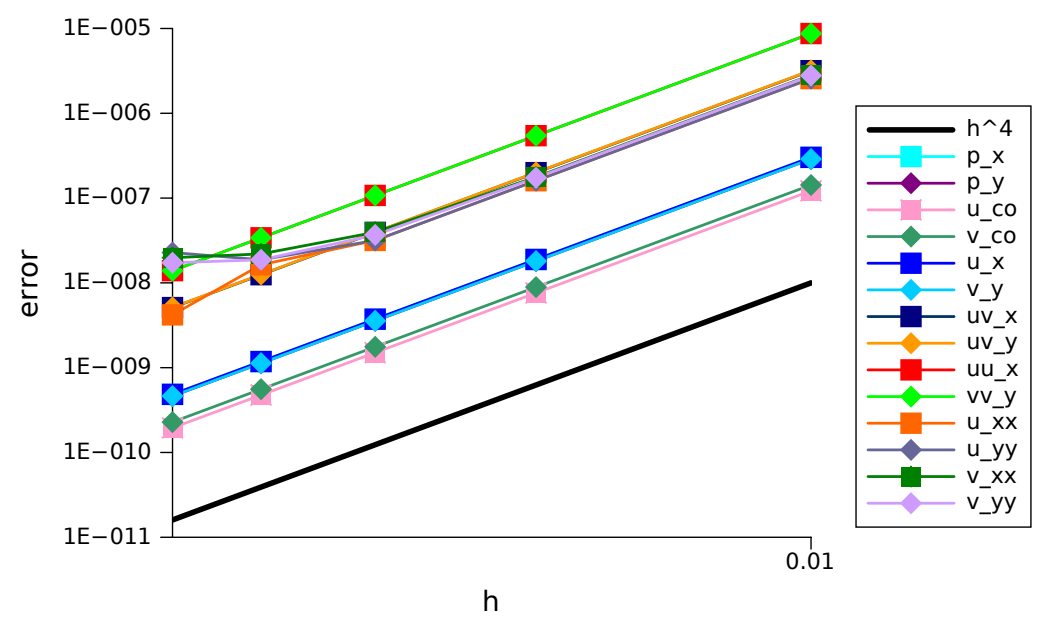

Figura 3: Gráfico $h \times \mid$ error $\left.\right|_{\infty}$. Análise de convergência no cálculo das derivadas. Os pontos correspondem a $h=1 / 100,1 / 200,1 / 300,1 / 400$ e $1 / 500$. Os subscritos em $\phi_{x}, \phi_{x x}$ e $\phi_{c o}$ referemse, respectivamente, à primeira derivada, à segunda derivada e às interpolações das velocidades para o canto da célula. Essa última é necessária para o cálculo do termo convectivo.

\subsection{Escoamento em uma cavidade}

O problema clássico de escoamento laminar dentro de uma cavidade quadrada na qual a tampa se move é simulado. Uma solução analítica conhecida é dada por [7]. O modelo matemático consiste das equações de Navier-Stokes com algumas simplificações: estado estacionário, escoamento laminar bidimensional nas direções $x$ e $y$, fluido incompressível. As condições de contorno para as velocidades $u$ e $v$ são do tipo Dirichlet: sendo nula em todos os pontos, exceto em $y=1$, onde temos

$$
u(x, 1)=16\left(x^{4}-2 x^{3}+x^{2}\right) .
$$


Um termo fonte $S$ está presente somente na equação da quantidade de movimento na direção $y$ e é dado como em [7]:

$$
S=-8 \nu\left[24 F(x)+2 f^{(1)}(x) g^{(2)}(y)+f^{(3)}(x) g(y)\right]-64\left[F_{2}(x) G_{1}(y)-g(y) g^{(1)}(y) F_{1}(x)\right],
$$

onde

$$
\begin{aligned}
f(x) & =x^{4}-2 x^{3}+x^{2} \\
g(y) & =y^{4}-y^{2} \\
F(x) & =\int f(x) d x=0.2 x^{5}-0.5 x^{4}+x^{3} / 3 \\
F_{1}(x) & =f(x) f^{(2)}(x)-\left[f^{(1)}(x)\right]^{2}=-4 x^{6}+12 x^{5}-14 x^{4}+8 x^{3}-2 x^{2}, \\
F_{2}(x) & =\int f(x) f^{(1)}(x) d x=0.5[f(x)]^{2}, \\
G_{1}(y) & =g(y) g^{(3)}(y)-g^{(1)}(y) g^{(2)}(y)=-24 y^{5}+8 y^{3}-4 y .
\end{aligned}
$$

A solução analítica é dada por

$$
\begin{aligned}
& u(x, y)=8 f(x) g^{(1)}(y)=8\left(x^{4}-2 x^{3}+x^{2}\right)\left(4 y^{3}-2 y\right), \\
& v(x, y)=-8 f^{(1)}(x) g(y)=-8\left(4 x^{3}-6 x^{2}+2 x\right)\left(y^{4}-y^{2}\right), \\
& p(x, y)=8 \nu\left[F(x) g^{(3)}(y)+f^{(1)}(x) g^{(1)}(y)\right]+64 F_{2}(x)\left\{g(y) g^{(2)}(y)-\left[g^{(1)}(y)\right]^{2}\right\} .
\end{aligned}
$$

A Figura 4 e a Tabela 1 apresentam os resultados, considerando: $\nu=1, \Delta t=10^{-6}$. Observe na figura que o campo de velocidades é semelhante ao problema clássico, no qual a tampa se move com velocidade constante igual a 1. A análise de convergência é observada na tabela. Apesar de os cálculos das derivadas feitos separadamente apresentarem quarta ordem, o método como um todo aparenta se aproximar com terceira ordem de precisão. Além disso, para esse problema, a interface foi colocada muito próxima aos pontos de malha (distância de $10^{-10}$ ao invés de $\left.\frac{1}{3} h\right)$.

Esses são, no entanto, resultados iniciais. Espera-se melhorar o método de forma que apresente quarta ordem para qualquer posicionamento da interface.

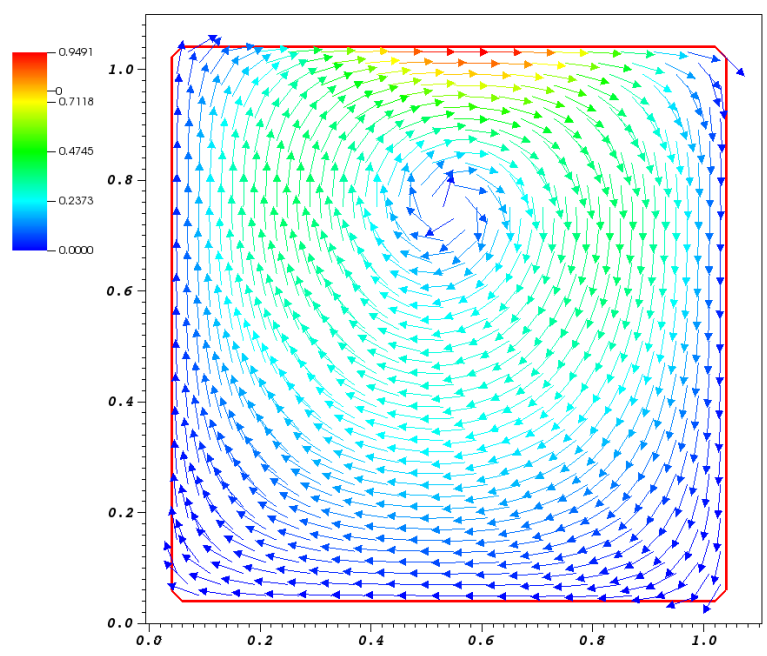

Figura 4: Escoamento em uma cavidade. Campo de velocidades. $\nu=1, \Delta t=10^{-6}$.

\section{Conclusões}

Foi apresentado o método das interfaces imersas implícito (MII-I), uma adaptação do método das interfaces imersas explícito (MII-E) desenvolvido por [4], que permite a simulação de escoamento de fluidos em geometrias complexas. Os resultados, ainda preliminares, são promissores. Foi obtida quarta ordem de aproximação para cálculo de derivadas e para a solução da equação de Poisson. No entanto, para a resolução das equações de Navier-Stokes por meio do método 
Proceeding Series of the Brazilian Society of Applied and Computational Mathematics, Vol. 3, N. 1, 2015.

\begin{tabular}{|c|c|c|c|c|c|c|}
\hline \multirow[t]{2}{*}{ Malha } & \multicolumn{2}{|c|}{ Norma $L_{1}$} & \multicolumn{2}{|c|}{ Norma $L_{2}$} & \multicolumn{2}{|c|}{ Norma $L_{\infty}$} \\
\hline & erro & ordem & erro & ordem & erro & ordem \\
\hline & \multicolumn{6}{|c|}{ velocidade $u$} \\
\hline $50 \times 50$ & $2.036261 \mathrm{e}-06$ & - & $2.541972 \mathrm{e}-06$ & - & $8.457417 \mathrm{e}-06$ & - \\
\hline $100 \times 100$ & $2.599824 \mathrm{e}-07$ & 2.9694 & $3.274744 \mathrm{e}-07$ & 2.9565 & $1.205539 \mathrm{e}-06$ & 2.8105 \\
\hline $150 \times 150$ & $7.759571 \mathrm{e}-08$ & 2.9820 & $9.813933 \mathrm{e}-08$ & 2.9719 & $3.890900 \mathrm{e}-07$ & 2.7891 \\
\hline \multirow[t]{2}{*}{$200 \times 200$} & $3.285584 \mathrm{e}-08$ & 2.9873 & $4.165165 \mathrm{e}-08$ & 2.9791 & $1.825710 \mathrm{e}-07$ & 2.6302 \\
\hline & \multicolumn{6}{|c|}{ velocidade $v$} \\
\hline $50 \times 50$ & $1.132684 \mathrm{e}-06$ & - & $1.959281 \mathrm{e}-06$ & - & $1.104794 \mathrm{e}-05$ & - \\
\hline 100x100 & $1.456096 \mathrm{e}-07$ & 2.9596 & $2.581196 \mathrm{e}-07$ & 2.9242 & $1.664289 \mathrm{e}-06$ & 2.7308 \\
\hline $150 \times 150$ & $4.355497 \mathrm{e}-08$ & 2.9766 & $7.795950 \mathrm{e}-08$ & 2.9527 & $5.349789 \mathrm{e}-07$ & 2.7991 \\
\hline $200 \times 200$ & $1.846175 \mathrm{e}-08$ & 2.9836 & $3.321987 \mathrm{e}-08$ & 2.9652 & $2.396510 \mathrm{e}-07$ & 2.7914 \\
\hline
\end{tabular}

Tabela 1: Erro e ordem de convergência.

da projeção, ainda há obstáculos que impedem a obtenção de quarta ordem. Esses obstáculos provavelmente estão relacionados com o método da projeção em si, em particular com a resolução da equação de Poisson. Pretende-se investigar formas alternativas de se resolver a equação de Poisson e também outras formulações do método da projeção.

\section{Agradecimentos}

Pelo apoio financeiro, processos n⿳02012/04471-5 e nº 2013/21501-8 Fundação de Amparo à Pesquisa do Estado de São Paulo (FAPESP).

\section{Referências}

[1] J. L. Guermond, L. Quartapelle, On stability and convergence of projection methods based on pressure poisson equation, I. J. Numerical Methods in Fluids, 26 (1998) 1039-1053.

[2] S. K. Lele, Compact finite difference schemes with spectral-like resolution, Journal of Computational Physics, 103 (1992) 16-42.

[3] R. J. LeVeque, Z. Li, The immersed interface method for elliptic equations with discontinuous coefficients and singular sources, SIAM J. Numerical Analysis, 31 (1994) 1019-1044.

[4] M. N. Linnick, H. F. Fasel, A high-order immersed interface method for simulating unsteady incompressible flows on irregular domains, J. Computational Physics, 204 (2005) 157-192.

[5] G. A. Reis, I. V. M. Tasso, L. F. Souza, J. A. Cuminato, "Fast and precise solution for the Poisson equation in the presence of interfaces with discontinuities", 22nd International Congress of Mechanical Engineering, Ribeirão Preto, 2013.

[6] C. J. Roy, Review of code and solution verification procedures for computational simulation, Journal of Computational Physics, 205 (2005) 131-156.

[7] T. Shih, C. Tan, B. Hwang, Effects of grid staggering on numerical schemes, International Journal for Numerical Methods in Fluids, 9 (1989) 193-212.

[8] D. A. von Terzi, M. N. Linnick, J. Seidel, H. F. Fasel, "Immersed boundary techniques for high-order finite-difference methods", AIAA Fluid Dynamics Conference and Exhibit, Anaheim, CA, 2001. 